\title{
Predictive Chromoendoscopy of Serrated Polyps: Is the Pendulum Swinging Toward the Pit Pattern?
}

\author{
Joseph C. Anderson ${ }^{1,3} \cdot$ Amitabh Srivastava $^{2}$ \\ Published online: 8 May 2018 \\ (c) This is a U.S. Government work and not under copyright protection in the US; foreign copyright protection may apply 2018
}

Endoscopic polypectomy is currently one of the most effective interventions for the prevention of colorectal cancer (CRC). Although the most common carcinoma precursor is the tubular adenoma, the detection, diagnosis, and follow-up of serrated precursors are also of clinical importance since the serrated pathway is implicated in about $30 \%$ of CRC [1, 2]. Serrated polyps can be categorized into three groups: the frequently encountered hyperplastic polyps (HPs) which are flat and distal; sessile serrated adenomas/polyps (SSA/Ps) which are flat, proximal, and account for about $10 \%$ of all serrated polyps; and traditional serrated adenomas (TSAs) which are distal, protruding, and account for a small percentage of serrated polyps [3]. HPs are further divided into microvesicular (MVHP) and goblet cell-rich (GCHP) types and are believed to be the precursors of SSA/Ps and TSAs, respectively. Although by definition SSA/Ps are non-dysplastic, they acquire cytologic dysplasia as they progress to CRC. Conversely, all TSAs harbor cytologic dysplasia. Thus, both SSA/P and TSA are established precursors of CRC. Detection and diagnosis of TSAs are straightforward given their unique endoscopic and histologic features. In contrast, HPs and SSA/Ps have similar endoscopic appearances and overlapping histologic features, complicating endoscopic and pathologic differentiation between the two lesions $[4,5]$. Furthermore, based on recent data, distinguishing SSA/Ps from HPs may also have some significance for metachronous

Disclaimer The contents of this work do not represent the views of the Department of Veterans Affairs or the United States Government.

Joseph C. Anderson

joseph.anderson@dartmouth.edu

1 Department of Veterans Affairs Medical Center, White River Junction, VT, USA

2 Brigham and Women's Hospital, Boston, MA, USA

3 The Geisel School of Medicine at Dartmouth, Hanover, NH, USA risk assessment [6], and therefore, differentiating HPs from SSA/Ps is a common concern for endoscopists.

A simple, accurate, and reproducible way to endoscopically distinguish HPs from SSA/Ps would aid endoscopists in their efforts to identify and remove all serrated lesions with malignant potential. Furthermore, these methods would be helpful in implementation of new paradigms in which diminutive polyps are optically diagnosed and either

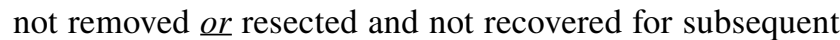
pathologic examination [7]. The American Society for Gastrointestinal Endoscopy (ASGE) Technology Committee's "Preservation and Incorporation of Valuable endoscopic Innovations" (PIVI) paper provides recommendations for adoption of new technologies or strategies into clinical practice, which can be used to optically diagnose diminutive $(\leq 5 \mathrm{~mm})$ polyps [8]. Although developed for adenomas, strategies for optical diagnosis can also be applied by endoscopists for serrated polyps. For example, use of the "diagnose and leave" strategy for serrated polyps could decrease the risk and cost of colonoscopy by obviating the need for polypectomy in polyps that were endoscopically diagnosed as HPs [9]. Alternatively, the "resect and discard" could decrease cost by eliminating the need for pathologic interpretation of serrated polyps that endoscopists were confident were HPs and not SSA/Ps.

In this month's issue of Digestive Diseases and Sciences [10], Aoki et al. in Sapporo, Japan, characterized serrated polyps, conventional adenomas, and CRC using endoscopic, pathologic, and molecular features. In addition to size and location, trained endoscopists used the Paris classification [11] to characterize the shape of the lesions. Magnification chromoendoscopy that has gained popularity in the Far East enables visualization of the "pit pattern" which reflects the colonic pit structure and enables the differentiation of the many types of polyps. The pit patterns are currently categorized by the Kudo classification $[12,13]$ where Type I indicates normal mucosa, Type II is consistent with HP, and Types III, IV, and V are consistent with dysplastic changes. 
The dysplastic pit patterns are commonly observed in conventional adenomas but may also exist in serrated polyps with dysplasia, including TSA and SSA/P with cytologic dysplasia. Type II pit patterns have recently been categorized further into Type II, Type II-O [14], and Type II-L. Although Type II may be predictive of HPs, recent data suggest that Type II-O [14] pit patterns may predict SSA/ Ps. The appearance of the Type II-O pattern is due to the basally dilated crypts that are characteristic of SSA/Ps. The significance, however, of Type II-L pattern is less clear, but perhaps predicts TSAs. All of the lesions in the current study were diagnosed by one experienced pathologist. The authors then used pyrosequencing to classify the lesions by mutational signature, proto-oncogene Kirsten rat sarcoma $(K R A S)$ or proto-oncogene B-Raf $(B R A F)$, the latter being closely linked with SSA/P-related CRC. They also used a panel of 5 methylation markers to separate polyps by $\mathrm{CpG}$ island methylation phenotype (CIMP) status into CIMP high, low, or negative. Table 1 presents proposed classifications for molecular, endoscopic, and pathologic features of serrated polyps.

How well did pit patterns predict final pathology in this study, specifically in differentiating HPs from SSA/Ps? In this analysis, $80 \%$ (80/100) of SSA/Ps had Type II-O, demonstrating moderate sensitivity. Furthermore, $88 \%$ (80/91) of all lesions with Type II-O patterns were SSA/P, suggesting that this pit pattern is predictive of SSA/Ps. Two of these lesions were SSA/Ps with dysplasia. Conversely, 69\% (33/48) of all Type II lesions were HPs but there were still $31 \%$ that were not HPs but mostly SSA/Ps and some TSAs or unclassified serrated polyps. Furthermore, about one-third of all HPs had a Type II-L or Type II-O pattern. When HPs were further divided into subcategories, nearly all of the HPs with Type II-O lesions were MVHPs as opposed to GCHPs, further lending credence to the idea that MVHP and SSA/P may be related. These data support previous findings that suggest that Type II-0 patterns may predict SSA/P.

With regard to distinguishing TSAs from HPs, the data regarding pit patterns were less convincing. Specifically, more than 50\% (8/15) of lesions with Type II-L were HPs; conversely, only a small proportion of TSAs had the Type II-L pattern. Most of the TSAs and other dysplastic serrated polyps exhibited Type III or IV patterns. A large proportion of serrated polyps with dysplasia $(83 \%$; 70/84) had Type III or IV appearance along with one of the three Type II pit patterns. Furthermore, the presence of any Type II pit pattern plus a Type III or IV indicated the presence of dysplasia in $\sim 80 \%(70 / 87)$ of serrated polyps.

How well did endoscopic pit patterns correlate with the polyp mutation profile? As would be predicted, most lesions (86\%; 78/91) with Type II-O had BRAF mutations, the oncogene that is closely linked with SSA/Ps. Along the same line, 94\% (34/36) of lesions with Type II-O plus III/IV also had
BRAF mutations. Conversely, Type II-L and Type II-L plus III/IV had a larger proportion of KRAS mutations, which is consistent with what one would expect for TSAs. The CIMP data appeared to be less helpful. For example, only half $(51 \%$; 47/91) of all Type II-O lesions were CIMP high, much less than what would be expected for SSA/Ps.

The investigators also examined the characteristics of Type II-L and Type II-O CRCs. The results here were more consistent with the current knowledge about the molecular the profile of serrated CRCs. Most of the Type II-O CRCs had BRAF mutations and Type II-L CRCs had KRAS mutations. Furthermore, most of the Type II-O CRCs were CIMP-H and had MLHI hypermethylation. Unfortunately, the small numbers of CRCs limited definite conclusions in the analysis.

The authors should be congratulated for undertaking this analysis that combined endoscopic, pathologic, and molecular characteristics to better understand and characterize serrated polyps. Furthermore, they analyzed a large group of serrated polyps, including rare lesions such as SSA/P with dysplasia and TSAs. The study also employed experienced endoscopists, crucial in order to accurately classify the shapes and pit patterns of the polyps. Furthermore, they examined the currently known molecular features of serrated polyps including $K R A S / B R A F$ mutations and the $\mathrm{CpG}$ island methylator phenotype.

The use of only one pathologist to interpret the slides is a major limitation since the histologic diagnoses were used as the 'gold standard' when examining the predictive values for the pit patterns. There were also issues with regard to the molecular markers chosen. Specifically, the methylation markers chosen to identify CIMP status are not specific for $B R A F$-mutated CIMP-H tumors. Although the authors chose to examine MINT1, MINT2, MINT12, MINT31, and p16 [15], the optimal genes to study CIMP-H versus CIMPL should have included RUNX3, CACNAIG, IGF2, MLH1, NEUROG1, GRABP1, SOCS1, and CDKN2A, since methylation of the first four genes is the most predictive of CIMP$\mathrm{H}$ lesions [15]. This likely explains the discordant results regarding CIMP status in the serrated polyps. Specifically, the $B R A F$-mutated lesions with Type II-O pattern, many of which were SSA/Ps, would be expected to show a higher prevalence of CIMP-H than was reported in the study. Other missed opportunities include failure to separate conventional dysplasia (mimicking tubular adenoma) and serrated dysplasia (mimicking TSA) in SSA/P. Examining MLH1 protein expression using immunohistochemistry in these serrated polyps and not just the cancers could have been helpful in this regard since most conventional cytologic dysplasia foci in SSA/P lose expression of MLH1 in contrast to serrated dysplasia in SSA/P that retain MLH1 expression. Moreover, recent observations show that some SSA/Ps do not progress to CRC through methylation of MLH1 and loss of the 
Table 1 Molecular, endoscopic, and histologic features of serrated and conventional polyps

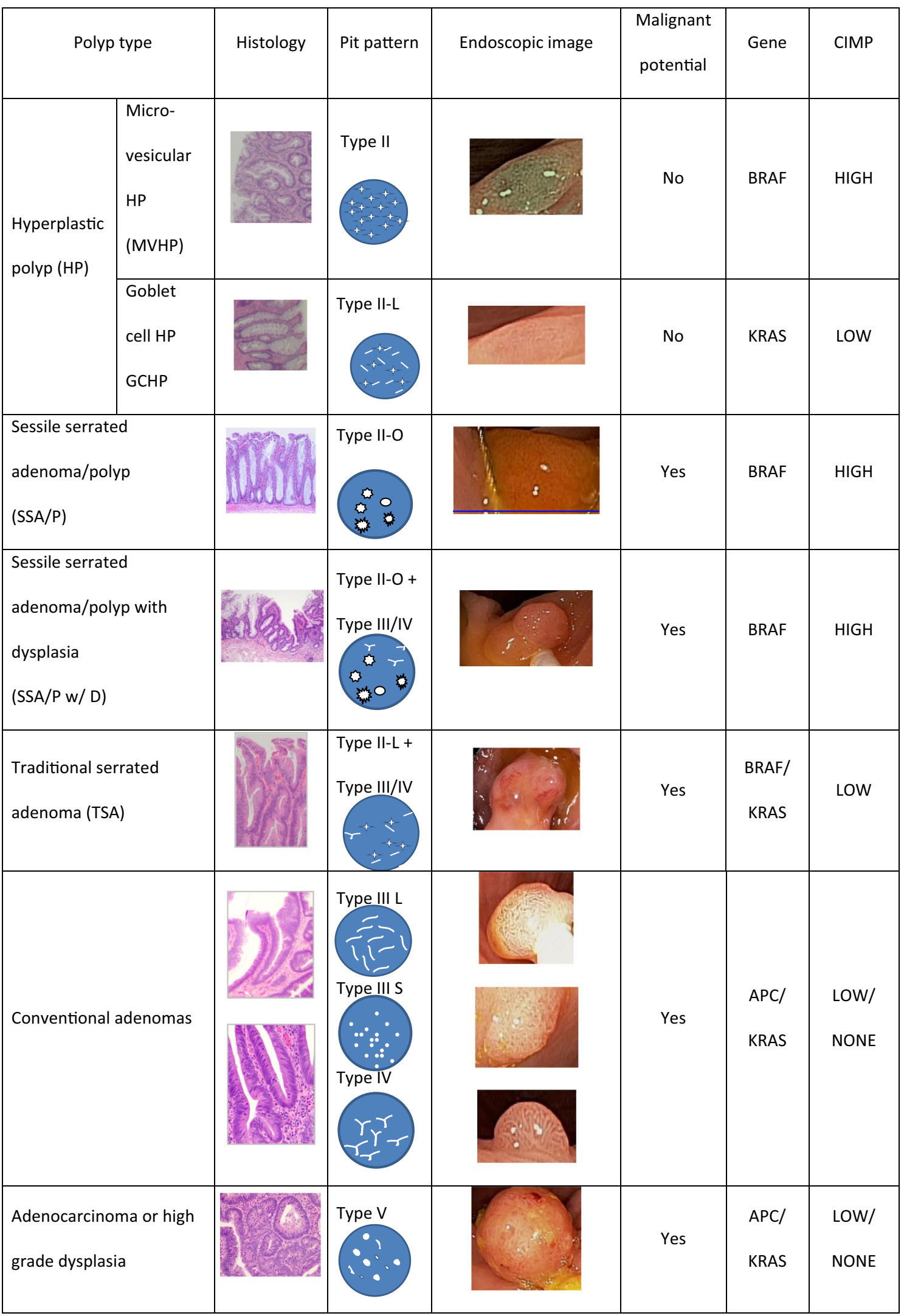

$A P C$ adenomatous polyposis coli, BRAF proto-oncogene B-Raf, CIMP CpG island methylator phenotype, KRAS proto-oncogene Kirsten rat sarcoma 
Fig. 1 Mucous cap on sessile serrated adenoma

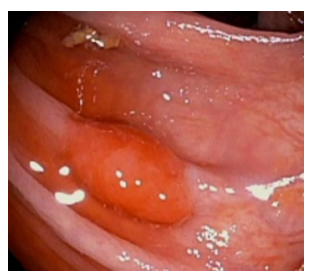

\section{References}

1. Anderson JC, Butterly LF, Goodrich M, et al. Differences in detection rates of adenomas and serrated polyps in screening versus surveillance colonoscopies, based on the new hampshire colonoscopy registry. Clin Gastroenterol Hepatol. 2013;11:1308-1312.

2. Anderson JC, Butterly LF, Weiss JE, et al. Providing data for serrated polyp detection rate benchmarks: an analysis of the New Hampshire Colonoscopy Registry. Gastrointest Endosc. 2017;85:1188-1194.

protein but instead progress through other mechanisms [16]. Thus, examining MLH1 status in the SSA/Ps might help to provide a better understanding of carcinogenesis as well as the meaning of certain endoscopic pit patterns in distinct subsets of dysplastic SSA/Ps.

The question for endoscopists is what place does recognizing pit patterns have in management of polyps? Certainly, the Type II-O pattern seems to be the most useful, predicting SSA/Ps almost $90 \%$ of the time. Conversely, Type II-L seems less helpful in distinguishing serrated polyps. Of note, ASGE PIVI recommends that for the "diagnose and leave" strategy, new technologies need to achieve a $90 \%$ negative predictive value (NPV) for adenomatous histology. This recommendation was developed for small adenomas, but the same criteria could be applied to serrated polyps. Unfortunately, the results observed in the current analysis for using the Type II-O pattern to distinguish SSA/Ps from HPs would not afford a $90 \%$ or greater NPV for SSA/Ps as suggested by PIVI-based recommendations. Another obstacle to implementation of the use of these pit patterns is that most Western endoscopists find the pit pattern classification relatively arcane as the technique is rarely taught in theory or in practice during fellowship in non-Asian programs. Still, with education, perhaps endoscopists could become more facile with this classification. One issue to address is whether the more predictive of the Type II patterns, Type II-O, could be further strengthened in predicting and optically diagnosing serrated polyps with the use of other clinical factors such as location, shape, and presence of the mucous cap (see Fig. 1). Furthermore, perhaps using other criteria such as the Workgroup on serrAted polypS and Polyposis (WASP) [17], which employ other endoscopic features of serrated polyps, might be useful in increasing the predictive ability of the pit patterns.

What are the next steps in using pit patterns to diagnose polyps, especially serrated lesions? One major limitation is that all studies that use histology as the gold standard are considered imperfect. Should future studies look at pit patterns and the metachronous risk of advanced adenomas and large serrated polyps without considering the histologic diagnosis? After all, if the aim is to reliably "resect and discard" or "diagnose and leave", then the endoscopic appearance is all that the gastroenterologists will have to rely on when assessing future risk of serrated polyps.
3. Anderson JC. Pathogenesis and management of serrated polyps: current status and future directions. Gut Liver. 2014;8:582-589.

4. Butterly L, Robinson CM, Anderson JC, et al. Serrated and adenomatous polyp detection increases with longer withdrawal time: results from the New Hampshire Colonoscopy Registry. Am J Gastroenterol. 2014;109:417-426.

5. Farris AB, Misdraji J, Srivastava A, et al. Sessile serrated adenoma: challenging discrimination from other serrated colonic polyps. Am J Surg Pathol. 2008;32:30-35.

6. Anderson JC, Butterly LF, Robinson CM, et al. Risk of metachronous high-risk adenomas and large serrated polyps in individuals with serrated polyps on index colonoscopy: data from the new hampshire colonoscopy registry. Gastroenterology. 2018;154:e2.

7. Abu Dayyeh BK, Thosani N, Konda V, et al. ASGE Technology Committee systematic review and meta-analysis assessing the ASGE PIVI thresholds for adopting real-time endoscopic assessment of the histology of diminutive colorectal polyps. Gastrointest Endosc. 2015;81:502 e1-502 e16.

8. Rex DK, Kahi C, O'Brien M, et al. The American Society for Gastrointestinal Endoscopy PIVI (Preservation and Incorporation of Valuable Endoscopic Innovations) on real-time endoscopic assessment of the histology of diminutive colorectal polyps. Gastrointest Endosc. 2011;73:419-422.

9. Hassan C, Pickhardt PJ, Rex DK. A resect and discard strategy would improve cost-effectiveness of colorectal cancer screening. Clinical Gastroenterology and Hepatology: the Official Clinical Practice Journal of the American Gastroenterological Association 2010;8:865-9, 869 e1-3.

10. Aoki H, Yamamoto E, Yamano H, et al. Subtypes of the Type II pit pattern reflect distinct molecular subclasses in the serrated neoplastic pathway. Dig Dis Sci. (Epub ahead of print). https:// doi.org/10.1007/s10620-018-5016-5.

11. Endoscopic Classification Review G. Update on the paris classification of superficial neoplastic lesions in the digestive tract. Endoscopy. 2005;37:570-578.

12. Kudo S, Lambert R, Allen JI, et al. Nonpolypoid neoplastic lesions of the colorectal mucosa. Gastrointest Endosc. 2008;68:S3-S47.

13. Kudo S, Tamura S, Nakajima T, et al. Diagnosis of colorectal tumorous lesions by magnifying endoscopy. Gastrointest Endosc. 1996;44:8-14.

14. Kimura T, Yamamoto E, Yamano HO, et al. A novel pit pattern identifies the precursor of colorectal cancer derived from sessile serrated adenoma. Am J Gastroenterol. 2012;107:460-469.

15. Weisenberger DJ, Siegmund KD, Campan M, et al. CpG island methylator phenotype underlies sporadic microsatellite instability and is tightly associated with BRAF mutation in colorectal cancer. Nat Genet. 2006;38:787-793.

16. Bettington M, Walker N, Rosty C, et al. Clinicopathological and molecular features of sessile serrated adenomas with dysplasia or carcinoma. Gut. 2017;66:97-106.

17. Je IJ, Bastiaansen BA, van Leerdam ME, et al. Development and validation of the WASP classification system for optical diagnosis of adenomas, hyperplastic polyps and sessile serrated adenomas/ polyps. Gut. 2016;65:963-970. 